\title{
Sentiment Analysis of Tweets using Heterogeneous Multi-layer Network Representation and Embedding
}

\author{
Loitongbam Gyanendro Singh, Anasua Mitra, Sanasam Ranbir Singh \\ Department of Computer Science and Engineering \\ Indian Institute of Technology Guwahati, Assam, India \\ \{gyanendrol9, anasua.mitra, ranbir\}eittg.ac.in
}

\begin{abstract}
Sentiment classification on tweets often needs to deal with the problems of under-specificity, noise, and multilingual content. This study proposes a heterogeneous multi-layer networkbased representation of tweets to generate multiple representations of a tweet and address the above issues. The generated representations are further ensembled and classified using a neural-based early fusion approach. Further, we propose a centrality aware random-walk for node embedding and tweet representations suitable for the multi-layer network. From various experimental analysis, it is evident that the proposed method can address the problem of under-specificity, noisy text, and multilingual content present in a tweet and provides better classification performance than the textbased counterparts. Further, the proposed centrality aware based random walk provides better representations than unbiased and other biased counterparts.
\end{abstract}

\section{Introduction}

With the growing popularity of Twitter, sentiment analysis of tweets has drawn the attention of several researchers from both academia and industry in recent times. Unlike other regular texts, sentiment analysis on Twitter text poses plenty of challenges because of various characteristics such as (i) under-specificity due to text limits, (ii) free-form writing such as the presence of user-defined hashtags, mentions, emoticons, (iii) noisy texts due to the presence of short-form, long-form, multilingual, transliterated text, misspelling. Researchers try to address these problems by adopting various methods like task-specific representation learning (Singh et al., 2020; Pham and Le, 2018; Fu et al., 2018; Tang et al., 2016; Kim, 2014), incorporating additional information such as hash-

\footnotetext{
${ }^{*}$ Equal contributions.
}

tags (Alfina et al., 2017; Qadir and Riloff, 2014), relationship between users (Zhao et al., 2017), multisource information (Zhou and Huang, 2017), ensembling (Al-Twairesh and Al-Negheimish, 2019; Araque et al., 2017; Wang et al., 2014), etc.

This paper proposes a novel approach to handle the above issues using a heterogeneous multi-layer network representation of a tweet. A multi-layer network is a network formulated by connecting different layers of networks. For example, a heterogeneous multi-layer network can be formed by connecting layers of networks of mentions, hashtags, and keywords. Multi-layer networks have shown to provide promising performance in other tasks like community detection and clustering (Hanteer and Rossi, 2019; Luo et al., 2020), node classification (Li et al., 2018; Zitnik and Leskovec, 2017; Ghorbani et al., 2019), representation learning in graphs (Cen et al., 2019; Zhang et al., 2018; Ni et al., 2018). A tweet or a collection of tweets can be represented by a multi-layer network. An advantage of using network-based representation is that a network can be expanded by adding nodes or shrunk by removing nodes. The motivations of using a multi-layer network in this paper are as follows. (i) The semantic relation between keywords, hashtags, and mentions can be captured by applying an effective network embedding method. (ii) The noise and under-specificity can be reduced by expanding the network with related nodes or by shrinking the network after removing the unrelated nodes. Further, the co-occurring keywords, hashtags, and mentions often share semantic relationships (Wang et al., 2016; Weston et al., 2014; Qadir and Riloff, 2013; Wang et al., 2011).

This paper has four major contributions. First, it transforms a tweet into a multi-layer network. Second, it proposes a centrality ${ }^{1}$ aware random

\footnotetext{
${ }^{1}$ Prominence of a node in a network
} 
walk over the multi-layer network. Third, it generates multiple representations of a tweet using the proposed centrality aware random walk and builds an early-fusion based neural sentiment classifier. Fourth, it also addresses under-specificity and noisy text for sentiment classification by expanding or shrinking the network representing the tweets. As such, sentiment classification is a domain-dependent task (Karamibekr and Ghorbani, 2012). Therefore, we evaluate the proposed method over datasets in different domains. From extensive experimental evaluations, the proposed method is found to outperform its counterparts in the majority of the cases. To the best of our knowledge, this study is the first of its kind to investigate sentiment classification task by transforming tweet into a heterogeneous multi-layer network.

The rest part of the paper is organized as follows. Section 2 presents the literature related to this study. Section 3 presents the proposed framework. The experimental setup is described in Section 4. The results and observations are analyzed in Section 5. Finally, Section 6 concludes the study of this paper.

\section{Related studies}

Sentiment analysis is an old research area. Initial work on sentiment classification can be traced back as early as 2000 (Turney, 2002; Pang et al., 2002; Turney and Littman, 2003). There have been several paradigm shifts in sentiment analysis methods from statistical methods (Turney, 2002; Pang et al., 2002; Turney and Littman, 2003) to rule-based (Prabowo and Thelwall, 2009), to lexicon-based (Taboada et al., 2011; Balamurali et al., 2011; Mohammad et al., 2009), to featurebased (Kouloumpis et al., 2011; Barbosa and Feng, 2010), to deep neural network (Kim, 2014; Severyn and Moschitti, 2015). Majority of the recent studies focus on the application of neural network models. Therefore, this section briefly reviews a few of the recent and related studies which have exploited graph and neural models.

Authors in (Violos et al., 2016) use a homogeneous network known as word graph to represent a document by connecting co-occurring words in the document. Three different networks are created for positive, negative, and neutral classes using the documents in respective classes. Using these networks, a document is represented by a threedimensional vector defined by the three sentiment classes. The elements of the vector correspond to the similarity of the word graph of the document and the word graph of the respective sentiment class. The vector thus obtained is used for classifying the document. Similarly, authors in (Bijari et al., 2020) construct co-occurrence word-graph of a document collection and generate word embedding using Node2 Vec (Grover and Leskovec, 2016). The embeddings thus obtained are used to represent words in the text and build a classifier using the Convolution Neural Network $(\mathrm{CNN})$ model. Further, in the studies (Gui et al., 2017; Zhao et al., 2017), the advantages of exploiting the relationship between keywords, sentiment, products and users have also been evident in sentiment analysis.

In recent times, deep learning based models are extensively used for sentiment classification. To mention few of them, authors in (Jianqiang et al., 2018; Dahou et al., 2016; Severyn and Moschitti, 2015; dos Santos and Gatti, 2014; Kim, 2014) use CNN, (Xu et al., 2019; Liu and Zhang, 2017) use Long Short Term Memory (LSTM), Bidirectional LSTM (Bi-LSTM), (Nguyen and Nguyen, 2018; Chen et al., 2017) use a combination of convolution and recurrent based neural network models. Further, studies (Al-Twairesh and Al-Negheimish, 2019; Araque et al., 2017) use neural ensemble models to combine different representation of text.

\section{Proposed framework}

As mentioned earlier, the proposed method has four distinct components; (i) representation of a tweet or collection of a tweet using a multi-layer network, (ii) centrality aware random walk over the multi-layer network, (iii) tweet classification using multiple representations generated from the multilayer network of a tweet, and (iv) reduction of noise in a tweet by expanding or shrinking network. This section discusses the details of these components. Figure 1 shows a high-level schematic diagram of the proposed model using a heterogeneous multilayer network.

\subsection{Representation of tweets using multi-layer network}

A $L$-layer network $\mathbf{G}$ is defined by $(\mathbf{V}, \mathbf{E}, \mathcal{L})$ where $\mathcal{L}$ denotes the set of layer indices $\{1,2, \ldots, L\}, \mathbf{V}=\left\{\mathbf{V}^{1} \cup \mathbf{V}^{2} \cup \ldots \cup \mathbf{V}^{L}\right\}, \mathbf{V}^{i}$ denotes the set of vertices in layer $i$ of the network, $\mathbf{E}$ denotes the set of edges. Considering three important components of a tweet, the proposed multi-layer network is formed with three layers 


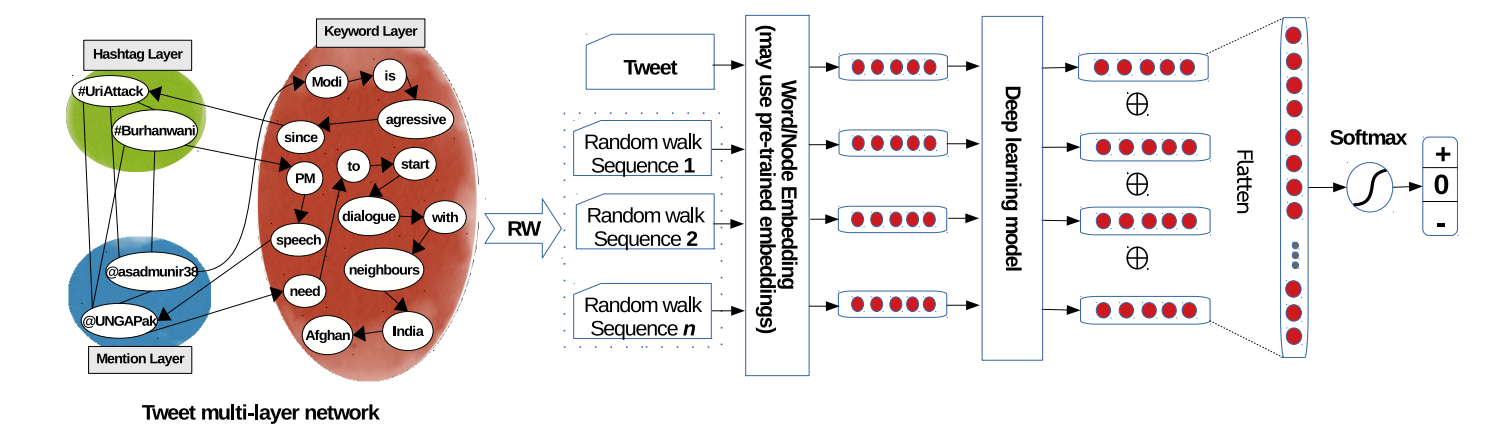

Tweet:@asadmunir38 Modi is agressive since \#UriAttack, \#BurhanWani \& PM speech @ UNGAPak needs to start dialogue with neighbours India, Afghan

Figure 1: Proposed heterogeneous multi-layer network based tweet sentiment classification framework

i.e., hashtag, mention and keyword as $\{H, M, K\}$. To capture both the co-occurrence and sequential characteristics of keywords, hashtags and mentions in a tweet, the proposed network consists of both directed and undirected edges. An edge $e_{x, y} \in \mathbf{E}$ is directed if $x$ and $y$ occur sequentially next to other in a tweet where, i) $x, y \in V^{K}$ or ii) $x \in V^{K}$ and $y \in\left\{V^{H} \cup V^{M}\right\}$ or iii) $x \in\left\{V^{H} \cup V^{M}\right\}$ and $y \in V^{K}$. Whereas, an edge $e_{x, y} \in \mathbf{E}$ is undirected if $x, y \in\left\{V^{H} \cup V^{M}\right\}$ co-occur in a tweet. An example of the proposed multi layer network for the tweet "@asadmunir38 Modi is agressive since \#UriAttack, \#BurhanWani \&PM speech@UNGAPak needs to start dialogue with neighbours India, Afghan" is shown in Figure 1. Edge set $\mathbf{E}=\{\mathbf{A} \cup \mathbf{B}\}$ which comprises of a set of intra-layer adjacency matrices $\mathbf{A}=\left\{\mathbf{A}^{1}, \mathbf{A}^{2}, \ldots, \mathbf{A}^{L}\right\}$ with matrix $\mathbf{A}^{i} \in$ $\mathcal{R}^{N^{i} \times N^{i}}$ in each layer $i$. A set of bipartite matrices $\mathbf{B}^{i, j} \in \mathcal{R}^{N^{i} \times N^{j}}$ represents cross-layer association between layer $i$ and layer $j$. For our tweet multi-layer network, we have three layers $\mathbf{A}=$ $\left\{\mathbf{A}^{H}, \mathbf{A}^{M}, \mathbf{A}^{K}\right\}$ and five types of bipartite associations $\mathbf{B}=\left\{\mathbf{B}^{H M}, \mathbf{B}^{M K}, \mathbf{B}^{H K}, \mathbf{B}^{K M}, \mathbf{B}^{K H}\right\}$. This kind of complex networks can also be viewed as one flattened representation in form of supraadjacency matrix $S$, with total nodes $N=\left|\mathbf{V}^{H}\right|+$ $\left|\mathbf{V}^{M}\right|+\left|\mathbf{V}^{K}\right|$,

$$
\mathbf{S}_{N \times N}=\left[\begin{array}{ccc}
\mathbf{A}^{H} & \mathbf{B}^{H M} & \mathbf{B}^{H K} \\
\mathbf{B}^{M H} & \mathbf{A}^{M} & \mathbf{B}^{M K} \\
\mathbf{B}^{K H} & \mathbf{B}^{K M} & \mathbf{A}^{K}
\end{array}\right]
$$

The intra-layer associations As are on the maindiagonal, and the cross-layer connections $\mathbf{B}$ are on the off-diagonal elements of S. Further, $\mathbf{A}^{K}, \mathbf{B}^{H K}, \mathbf{B}^{K H}, \mathbf{B}^{M K}, \mathbf{B}^{K M}$ are asymmetric matrices and other matrices of $\mathbf{S}$ are symmetric. A tweet or a collection of tweets can be represented as a multi-layer network, as discussed above.

\subsection{Centrality aware random-walk with restart for heterogeneous multi-layer network}

To generate random walk sequences from the proposed multi-layer tweet network, we extend the random walk followed in PageRank (Brin and Page, 1998) algorithm. Given a row stochastic adjacency matrix A of a network, the PageRank of the nodes in the network can be defined as the following vector.

$$
\vec{\pi}_{t+1}=(1-\delta) \mathbf{A} \vec{\pi}_{t}+\delta \vec{\pi}_{0}
$$

where $\vec{\pi}_{t}$ is the stationary probability distribution vector that depicts the probability with which a random walker would stay in a particular node at time $t$. The restart probability $\delta \in[0,1]$ denotes the probability of jumping to a random node and $\vec{\pi}_{0}$ is the initial stationary probability vector.

As in (Li and Patra, 2010), the above randomwalk can be extended to our tweet multi-layer heterogeneous network in the following manner. If $\lambda \in(0,1)$ is the probability that a random-walker jumps to a different layer while surfing, in presence of $L$ number of layers and considering jumping to any of the remaining layers is equiprobable, the transition probability $\mathbf{M}$ aka column-normalized supra-adjacency matrix $\mathbf{S}$ in Equation 1, is modified as,

$$
\mathbf{M}=\left[\begin{array}{ccc}
(1-\lambda) A^{H} & \frac{\lambda}{L-1} B^{H M} & \frac{\lambda}{L-1} B^{H K} \\
\frac{\lambda}{L-1} B^{M H} & (1-\lambda) A^{M} & \frac{\lambda}{L-1} B^{M K} \\
\frac{\lambda}{L-1} B^{K H} & \frac{\lambda}{L-1} B^{K M} & (1-\lambda) A^{K}
\end{array}\right]
$$

That is, for a node, if its bipartite association exists, a random-surfer can stay in the same layer with probability $(1-\lambda)$ or transit to a different layer with probability $\left(\frac{\lambda}{L-1}\right)$. Now, Equation 2 can be re-written as follows,

$$
\vec{\pi}_{t+1}=(1-\delta) \mathbf{M} \vec{\pi}_{t}+\delta \vec{\pi}_{r s}
$$


where $\vec{\pi}_{r s}=\left[\begin{array}{c}\eta_{H} \cdot \vec{\pi}_{0}^{H} \\ \eta_{M} \cdot \vec{\pi}_{0}^{M} \\ \eta_{K} \cdot \vec{\pi}_{0}^{K}\end{array}\right], \eta_{i}$ denotes the importance of layer $i, \vec{\pi}_{0}^{i}$ denotes the initial stationary distribution of nodes in layer $i$ and $\sum_{i \in\{H, M, K\}} \eta_{i}=$ 1. And, $\vec{\pi}_{t} \in \mathcal{R}^{\left(N^{H}+N^{M}+N^{K}\right)}$ is the stationary probability distribution of a random surfer on the heterogeneous multi-layer network at time $t$.

In this study, we propose to personalize the above PageRank algorithm using the global importance of nodes in the proposed heterogeneous multi-layer network. In Equation 4, $\vec{\pi}_{r s}$ the restart probability vector is interpreted as layer importance weighted over the centrality based initial stationary probabilities of nodes. This interpretation needs not only the node centrality scores but also the layer importances. MultiRank (Rahmede et al., 2018), a centrality estimate for multiplex networks ${ }^{2}$ formulated using a modified version of PageRank algorithm, can estimate both the node centrality scores as well as the layer influences. MultiRank uses a layer-influence weighted aggregated adjacency matrix and a weighted bipartite matrix that relates nodes with layers to determine the node and layer centrality scores simultaneously. We specifically change the definition of these two matrices to customize the MultiRank algorithm for estimating the centrality scores over the heterogeneous multi-layer network representation of tweets. As we calculate the centrality scores, we modify $\vec{\pi}_{r s}$ of Equation 4 by replacing each $\eta_{i}$ with respective influence score of layer $i$ and each initial stationary vector $\vec{\pi}_{0}^{i}$ with node centrality scores in layer $i$.

In the customized MultiRank algorithm, we have tuned free-parameters (as described in the original paper) while calculating the centrality scores - i) to suppress or enhance the contribution of lowcentrality nodes, ii) to take into account the elite layers that contain a few highly central nodes, iii) to or not to normalize layer influences by weighted layer in-strength. We have tuned the restart parameter in MultiRank and multi-layer random walks in the range $\in[0.5,0.85]$. In this study, the MultiRank algorithm and multi-layer random walks gave the best performance by setting the restart parameter to 0.5 and 0.85 , respectively. Furthermore, the average number of tokens per tweet present in our training dataset is 29 , so we have hypothetically set

\footnotetext{
${ }^{2}$ Multiplex network (Kivelä et al., 2014) is a special case of a multi-layer network that has the same set of nodes exhibiting distinct relations in different layers.
}

\begin{tabular}{l} 
Node embedding methods \\
\hline \multicolumn{2}{|c|}{ FastText Embedding (FT) (Bojanowski et al., 2017) } \\
Multi-View Embedding (MVE) (Qu et al., 2017) \\
Multiplex Network Embedding (MNE) (Zhang et al., 2018) \\
Sentiment Hashtag Embedding (SHE) (Singh et al., 2020) \\
* The embedding dimension is of 128 size. Same hyper-parameter as suggested in the literature. \\
\begin{tabular}{|l|l|}
\hline \multicolumn{1}{|c|}{ Heep-learning models } & \multicolumn{1}{|c|}{ Hyper-parameter } \\
\hline $\begin{array}{l}\text { Convolution Neural Network } \\
\text { (CNN) }\end{array}$ & 3 Kernels, 128 \#Filters, ReLu \\
\hline Bidirectional Long Short & Activation Function \\
Term Memory (Bi-LSTM) & tion Function \\
\hline
\end{tabular}
\end{tabular}

Table 1: Different embedding and neural methods

the walk-length at 30 . We set the number of walks at 10. All the free parameters are tuned based on end-task performance.

\subsection{Classification of tweets represented with a multi-layer network}

Let $G_{i}$ be the multi-layer network representing a tweet $T_{i}$. Over this network, we generate $n$ number of node sequences $\mathcal{S}=\left\{\mathcal{S}_{1}, \mathcal{S}_{2}, \ldots, \mathcal{S}_{n}\right\}$ by using the above proposed random walk. Each node sequence is maintained to have a length of $m$ nodes. With $n$ number of random sequences and the original tweet, we have $(n+1)$ sentences to represent the tweet $T_{i}$. Each word in these sentences can be represented using a vector obtained from an appropriate embedding method. This paper has considered different embedding methods, as listed in Table 1, trained over a large collection of tweets.

For each node sequence $\mathcal{S}_{i}$, we apply a neural model (Bi-LSTM (Chen et al., 2017) and CNN (Kim, 2014)) to generate a representation of the sequence $\mathcal{S}_{i}$. The last hidden state output obtained after passing the node sequences to $\mathrm{Bi}$ LSTM represents the sequence $\mathcal{S}_{i}$. While, the vector obtained after applying the pooling step in CNN represents the sequence $\mathcal{S}_{i}$. Thus, we obtained $(n+1)$ vectors for each tweet. We concatenate these $(n+1)$ vectors and feed it to a feed-forward dense layer with three neurons (each for positive, negative, and neutral) and classify the sentiment of the tweet using softmax activation function in the output layer as shown in Figure 1. We use Keras ${ }^{3}$ deep learning framework for building our proposed model.

We calculate the error loss $(\Delta)$ for the classifier using the well-known cross-entropy loss as,

$$
\Delta=-\frac{1}{l} \sum_{i=1}^{l} \sum_{c} \mathbf{t}_{i c} \log \left(s_{i c}\right)
$$


where $c$ is the number of sentiment classes, $\mathbf{t}_{i c}$ is the $c^{\text {th }}$ ground truth class for the tweet, $l$ is the total number of training samples, and $\mathbf{s}_{i c}$ is the predicted probability on sample $i$ for the $c^{\text {th }}$ class.

\subsection{Network expansion and shrinking}

One of the motivations of using the multi-layer network for representing a tweet lies in its flexibility to expand or shrink the network. Given a set of existing nodes in a tweet-network as query nodes, the idea is to identify the most related nodes or most noisy nodes by exploiting a multi-layer network of a global tweet collection. We consider the most central and most similar neighboring nodes of the query nodes as potential expansion candidates. To reduce the search space, we first select the top $k$ query nodes ranked by the nodes' centrality scores in the tweet network view. The centrality scores of the nodes are calculated from the whole tweets collection. We then find neighbors of the selected nodes and ranked them using a weighted combination of similarity and centrality score using the scoring function defined below: $\operatorname{Score}(v)=\sum_{u \in N_{v}} \alpha \cdot \operatorname{sim}(v, u)+(1-\alpha) \cdot \operatorname{centrality}(u)$ where $N_{v}$ denotes neighbouring nodes of $v$, $\operatorname{sim}(v, u)$ denotes cosine similarity using node embeddings of $v$ and $u$, and centrality $(u)$ denotes centrality score of node $u$ in global network. In this study, we take equal weights of cosine and centrality score by setting $\alpha=0.5$. Top neighbouring nodes are selected using the above scoring function and added to the network in their respective layers using the edge policy discussed in Section 3.1.

The above node expansion method finds new nodes having semantic relation with the query nodes. However, for the sentiment analysis task, we are interested in adding only sentiment bearing nodes by selecting only those nodes having the dominant sentiment class among the selected nodes for expansion. While, the rest of the nodes with less dominating sentiment classes are removed from the tweet network. The Sentiment Hashtag Embedding (SHE) method proposed in (Singh et al., 2020) is used to estimate the sentiment orientation of a node. We have used the same experimental setup as described in the literature.

\begin{tabular}{|c|c|c|c|}
\hline \multicolumn{4}{|c|}{ Heterogeneous Multi-layer Tweet Network } \\
\hline Relation & \#Nodes & \#Edges* & Edge-type \\
\hline Hashtag-Hashtag, $A^{H}$ & 3552 & 10776 & Undirected \\
\hline Mention-Mention, $A^{M}$ & 4243 & 12277 & Undirected \\
\hline Keyword-Keyword, $A^{K}$ & 28962 & 181849 & Directed \\
\hline Hashtag-Mention, $B^{H M}$ & 6446 & 13765 & Undirected \\
\hline Hashtag-Keyword, $B^{H K}$ & 4782 & 6648 & Directed \\
\hline Mention-Keyword, $B^{M K}$ & 7958 & 14790 & Directed \\
\hline Keyword-Hashtag, $B^{K H}$ & 6824 & 11825 & Directed \\
\hline Keyword-Mention, $B^{K M}$ & 4018 & 5813 & Directed \\
\hline \multirow{2}{*}{\multicolumn{4}{|c|}{$*^{*}$ The edges are weighted by normalized co-occurrence frequency. }} \\
\hline & & & \\
\hline \#Positive & \#Negative & \#Neutral & Total Tweets \\
\hline Societal & 17047 & 9000 & 42422 \\
\hline
\end{tabular}

Table 2: Statistical characteristics of the dataset

\section{Experimental Setup}

\subsection{Dataset}

This paper considers a locally annotated dataset named as Societal. We have collected 50,300 tweets using Twitter Streaming $\mathrm{API}^{4}$ over four events that happened in India during AugustDecember 2016, namely Uri Attack, Surgical Strike, GST Amendment Bill, and Demonetization. Two annotators with strong command on English and Hindi are engaged to annotate the tweets with positive, negative, and neutral sentiments. We have selected 42,422 tweets where the two annotators have agreed on the same sentiment, which is of $85 \%$ agreement having 82.35 Kappa coefficient scores. The majority of the disagreements among the annotators are on the tweets with stance and sarcastic natures. A similar observation is also reported in (Karamibekr and Ghorbani, 2012). The Societal dataset contains $18 \%$ non-English tweets (i.e., Hindi and code-mix with English), of which 1,626 code-mix tweets and 1,505 tweets with less than five keywords are kept unseen for evaluation of our proposed model. Meanwhile, the hashtags and mentions cover $11 \%$ and $15 \%$ of the total 39,428 unique vocabulary of the Societal dataset. This dataset is used to build sentiment classifiers and construct a multi-layer network to generate node embeddings. Details of the dataset is shown in Table 2.

\subsection{Embedding method}

We investigate the efficacy of our proposed multilayer network using four different types of node embedding methods namely Multiplex Network Embedding (MNE) (Zhang et al., 2018), MultiView Embedding (MVE) (Qu et al., 2017), FastText (FT) (Bojanowski et al., 2017), and Sentiment Hashtag Embedding (SHE) (Singh et al., 2020)

\footnotetext{
$4_{\text {http: }: / / \text { docs.tweepy. org }}$
} 


\begin{tabular}{|c|c|c|c|c|c|c|c|c|c|c|c|c|c|c|c|c|c|}
\hline \multicolumn{2}{|c|}{ Types of tweet representation } & \multicolumn{8}{|c|}{ Accuracy (in \%) } & \multicolumn{8}{|c|}{ F-Macro (in \%) } \\
\hline & RW & BFT & MNE & MVE & SHE & BFT & MNE & MVE & SHE & BFT & MNE & MVE & SHE & BFT & MNE & MVE & SHE \\
\hline Original Tweet & - & 77.92 & 75.53 & 77.01 & 76.89 & 75.22 & 74.53 & 73.64 & 76.05 & 76.62 & 73.52 & 75.33 & 75.38 & 72.43 & 72.59 & 71.60 & 74.39 \\
\hline [A] T+MLN & $\begin{array}{c}\text { Unbiased } \\
\text { N2V } \\
\text { Biased }\end{array}$ & $\begin{array}{l}73.96 \\
75.61 \\
77.88\end{array}$ & $\begin{array}{l}74.90 \\
75.45 \\
74.30\end{array}$ & $\begin{array}{l}75.10 \\
75.02 \\
74.39\end{array}$ & $\begin{array}{l}76.51 \\
74.15 \\
77.27\end{array}$ & $\begin{array}{l}74.83 \\
74.65 \\
75.89\end{array}$ & $\begin{array}{l}74.38 \\
72.57 \\
74.70\end{array}$ & $\begin{array}{l}73.90 \\
72.84 \\
74.37\end{array}$ & $\begin{array}{l}75.70 \\
73.84 \\
75.63\end{array}$ & $\begin{array}{l}70.99 \\
72.56 \\
75.07\end{array}$ & $\begin{array}{l}72.14 \\
73.03 \\
71.34\end{array}$ & $\begin{array}{l}72.68 \\
72.83 \\
72.83\end{array}$ & $\begin{array}{l}73.49 \\
71.68 \\
74.85\end{array}$ & $\begin{array}{l}71.88 \\
72.09 \\
73.35\end{array}$ & $\begin{array}{l}72.62 \\
70.51 \\
72.58\end{array}$ & $\begin{array}{l}71.69 \\
70.70 \\
72.73\end{array}$ & $\begin{array}{l}72.67 \\
70.82 \\
73.00\end{array}$ \\
\hline [B] T+MLN+NE & $\begin{array}{c}\text { Unbiased } \\
\text { N2V } \\
\text { Biased }\end{array}$ & $\begin{array}{l}776.20 \\
75.30 \\
78.33\end{array}$ & $\begin{array}{l}75.30 \\
73.80 \\
76.57\end{array}$ & $\begin{array}{l}75.08 \\
72.67 \\
76.54\end{array}$ & $\begin{array}{l}77.18 \\
73.84 \\
77.88\end{array}$ & $\begin{array}{l}75.31 \\
74.54 \\
76.33\end{array}$ & $\begin{array}{l}74.96 \\
74.77 \\
75.08\end{array}$ & $\begin{array}{l}74.53 \\
72.49 \\
75.05\end{array}$ & $\begin{array}{l}75.51 \\
73.84 \\
76.53\end{array}$ & $\begin{array}{l}73.85 \\
72.46 \\
76.84\end{array}$ & $\begin{array}{l}72.93 \\
71.47 \\
74.15\end{array}$ & $\begin{array}{l}73.04 \\
70.91 \\
73.01\end{array}$ & $\begin{array}{l}74.48 \\
72.13 \\
75.05\end{array}$ & $\begin{array}{l}72.87 \\
72.25 \\
74.92\end{array}$ & $\begin{array}{l}71.63 \\
72.50 \\
73.41\end{array}$ & $\begin{array}{l}72.17 \\
70.75 \\
73.32\end{array}$ & $\begin{array}{l}73.08 \\
71.85 \\
74.44\end{array}$ \\
\hline [C] $\mathbf{T}+\mathbf{M L N}+\mathbf{S N E}$ & $\begin{array}{l}\text { Unbiased } \\
\text { N2V } \\
\text { Biased }\end{array}$ & $\begin{array}{l}78.72 \\
77.77 \\
79.23\end{array}$ & $\begin{array}{l}76.20 \\
76.66 \\
77.97\end{array}$ & $\begin{array}{l}77.17 \\
77.38 \\
78.14\end{array}$ & $\begin{array}{l}79.37 \\
76.87 \\
80.78\end{array}$ & $\begin{array}{l}76.97 \\
76.72 \\
78.95\end{array}$ & $\begin{array}{l}74.87 \\
72.45 \\
77.11\end{array}$ & $\begin{array}{l}75.73 \\
76.47 \\
78.16\end{array}$ & $\begin{array}{l}76.79 \\
76.11 \\
79.33\end{array}$ & $\begin{array}{l}777.39 \\
76.68 \\
77.33\end{array}$ & $\begin{array}{l}76.43 \\
75.50 \\
76.73\end{array}$ & $\begin{array}{l}75.52 \\
76.13 \\
76.90\end{array}$ & $\begin{array}{l}78.09 \\
74.65 \\
79.79\end{array}$ & $\begin{array}{l}75.73 \\
75.30 \\
77.39\end{array}$ & $\begin{array}{l}73.08 \\
70.86 \\
75.79\end{array}$ & $\begin{array}{l}74.32 \\
73.41 \\
76.66\end{array}$ & $\begin{array}{l}73.84 \\
73.69 \\
78.22\end{array}$ \\
\hline [D] $\mathbf{T}+$ Shuffle & $\begin{array}{l}\text { Unfiltered } \\
\text { Filtered }\end{array}$ & $\begin{array}{l}73.86 \\
77.54\end{array}$ & $\begin{array}{l}76.66 \\
77.17\end{array}$ & $\begin{array}{l}76.26 \\
77.84\end{array}$ & $\begin{array}{l}77.49 \\
77.89\end{array}$ & $\begin{array}{l}74.98 \\
76.21\end{array}$ & $\begin{array}{l}75.05 \\
76.84\end{array}$ & $\begin{array}{l}76.26 \\
76.98\end{array}$ & $\begin{array}{l}76.33 \\
77.78\end{array}$ & $\begin{array}{l}73.04 \\
76.48\end{array}$ & $\begin{array}{l}75.15 \\
75.95\end{array}$ & $\begin{array}{l}74.20 \\
76.43\end{array}$ & $\begin{array}{l}75.04 \\
75.07\end{array}$ & $\begin{array}{l}72.91 \\
75.07\end{array}$ & $\begin{array}{l}73.29 \\
75.32\end{array}$ & $\begin{array}{l}74.54 \\
75.18\end{array}$ & $\begin{array}{l}73.93 \\
76.17\end{array}$ \\
\hline
\end{tabular}

Table 3: Performance of sentiment classifiers across different embedding and representations. Blue: Embedding method that performs best for each tweet representations. Red: Best performing tweet representation for each embedding models. Purple: Best performing classifier across different representation of tweet and embedding models. Purple bold: Overall best.

(listed in Table 1). These embedding methods need a collection of node sequences. This study represents the tweet corpus into an expanded multilayer network by combining the whole tweet networks to generate node sequences via a random walk method. For experimental comparison, we investigate three random walk methods to generate the node sequences, namely Unbiased random walk used in MNE, biased random walk used in Node2Vec (N2V) (Grover and Leskovec, 2016) and the proposed centrality aware Biased random walk. Moreover, to investigate the efficacy of our proposed random walk (RW), we modeled the generated Biased RW sequences using the FastText embedding model - which we refer to as Biased FT (BFT) in Table 3.

\subsection{Selection of $n$ random walks}

A random walker can generate various node sequences starting from a node in the given network. However, all of the sequences are not useful. To identify the node sequences of our interest, we consider a simple second-order Markov chain based language model (Lafferty and Zhai, 2001) by calculating the probability of generating a node sequence given a tweet network. This study considers the top three random-walk sequences. ${ }^{5}$

\section{Results and observations}

In Table 3, we show the performance of two sentiment classifiers CNN (Nguyen and Nguyen, 2018) and Bi-LSTM (Xu et al., 2019) in terms of accuracy and F-Macro scores over the Societal dataset using 10-fold cross validation approach for four embedding models of our choice namely Multiplex Network Embedding (MNE) (Zhang et al.,

\footnotetext{
${ }^{5}$ We have considered only the top few walks $(3,5$, and 7$)$ with the highest probability. Experiments show that considering the top 3 walks provide the best results. The codes for this paper are available at: https://github. com/gloitongbam/SA_Hetero_Net
}

2018), Multi-View Embedding (MVE) (Qu et al., 2017), FastText (FT) (Bojanowski et al., 2017), and Sentiment Hashtag Embedding (SHE) (Singh et al., 2020). We consider the work of (Nguyen and Nguyen, 2018; Xu et al., 2019) as the baseline models for text-based sentiment classification of tweet. Along the rows of Table 3, we have three groups namely $[\mathrm{A}],[\mathrm{B}]$ and $[\mathrm{C}]$ pertaining to the three types of tweet-network representations, where we compare three different types of Random-Walks (RWs) - Unbiased, Node2Vec (N2V) and the proposed Biased RW to generate node sequences required as inputs for the above embedding methods. From the table, we can see that the network representation of tweets helps the sentiment classification task. Though the tweet-text only classification (in the first row) is hard to beat using the multilayer network representation of a tweet without node expansion, but for Bi-LSTM based classifier, the classifiers using Biased RW in the group [A] beats text only prediction in $75 \%$ of the cases with a maximum of $1.13 \%$ difference in terms of F-macro using Biased FT embeddings. For CNN classifier, the Biased RW in [A] beats original tweet prediction using SHE embeddings. Although the classifiers in [B] gave a competitive performance as compared to text-only classifiers in [A], sentiment polarized node expansion (SNE) method in $[\mathrm{C}]$ beats tweet-text based prediction by a margin of $1.4 \%$, and $1.9 \%$ (on average) for CNN and Bi-LSTM classifiers respectively - indicating the network representation of tweets, especially when augmented with informative nodes, are useful and complements the text in tweets. Among the RW based methods for node sequence generation, the proposed Biased RW performs the best followed by Unbiased and $N 2 V$. The proposed Biased RW outperforms Unbiased RW decently - can be seen with prominence in [A] Biased vs Unbiased RWs 
for CNN classifiers using Biased FT embedding. Even the best performances in both the metrics pertain to [C] Biased RW with SHE embedding using both the classifiers. We feel the $N 2 V$ style global topology-based biasing is not that useful for sentiment prediction than our biased approach, which uses centrality scores intuitively. Among the embedding models, we observe that Biased FT and SHE give competitive performances. We believe Biased FT performs competitively as it is trained on centrality-aware random-walks, additionally augmented with sentiment polarized nodes. Whereas, SHE systematically embeds sentiment information and also aided by biased tweet graph view - this makes it an unbeatable performer for sentiment classification.

To realize the importance of generating node sequences with an effective RW method over the proposed network, we investigate another experimental setup by randomly shuffling the selected nodes for expansion (both sentiment polarized and non-polarized nodes) with the tweet text. We call it as T+Shuffle-Filtered and Unfiltered methods for shuffling of sentiment polarized and nonpolarized node expansions respectively in [D]. For Bi-LSTM, we can see [D] Unfiltered beats textonly prediction, which signifies that the list of selected nodes, though randomly shuffled, but are informative enough. For both the classifiers in [D] Filtered outperforms text-only prediction on average by $0.8 \%, 2.4 \%$, respectively, signifying selected nodes by sentiment polarized node expansion method aids in performance. Here we shall also showcase the novelty of node sequences over a randomly shuffled list of the same nodes. [D] $U n$ filtered is comparable with [B] view - Biased RWs are seen to improve upon the prior. Whereas walks in the $[\mathrm{C}]$ view, which is comparable to [D] Filtered are seen to improve the performance of the latter. [C] Biased RW beats [D] Filtered by 1.6\%, 1.5\% points on average for CNN and Bi-LSTM.

\subsection{Novelty of centrality-aware walks}

It is evident from the already-shown results that our proposed biased random-walks are useful for the effective representation of tweets. One may be further interested in knowing how far these $B i$ ased RW sequences can improve any embedding models' performance. We conduct a pilot study by creating three versions of the FastText algorithm a word embedding based original version (FT), an

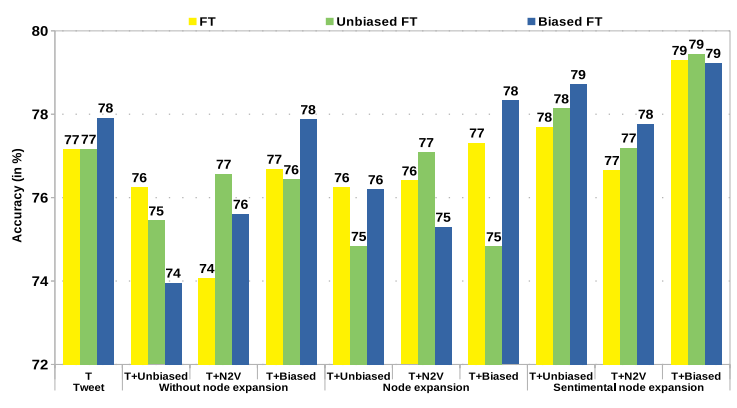

* The plot shows different scale but of same value due to round-off error.

Figure 2: Performance of CNN classifier using different types of node embedding generated via FastText algorithm

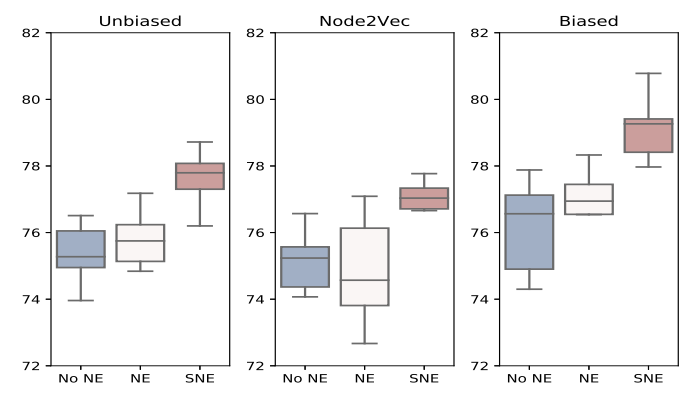

Figure 3: Effectiveness of (sentiment polarized) node expansion in tweet-network representations. A:Unbiased, B:Node2 Vec, C:Biased representation of tweet-network for No Node Expansion (No NE), Node Expansion (NE), sentiment polarized node expansion (SNE) methods. Accuracy(\%) of sentiment prediction is in Y-axis.

Unbiased RW sequence-based version (Unbiased FT), and a Biased RW sequence-based version (Biased FT) as summarized in Figure 2. Biased FT beats tweet-based FT in 6 out of 10 cases by an average of $1.11 \%$. Biased FT also beats Unbiased FT in $6 / 10$ cases by an average of $1.37 \%$. Although Unbiased FT seems to perform poorer as compared to the original FT in general, in the case of sentiment polarized node expansion, it consistently outperformed the FT - which again proves the effectiveness of the sentiment polarized node expansion method.

\subsection{Novelty of sentiment polarized node expansion}

In this section, we further analyzed the effectiveness of node expansion for the sentiment classification task. We summarize using box-plot in Figure 3 , the performances of the tweet-network representations (shown in Table 3) for sentiment polarized and non-polarized node expansion, and without node expansion over different RW algorithms (i.e. Unbiased, Node2Vec, Biased). From the figure, it is observed that for each RW methods, the node ex- 


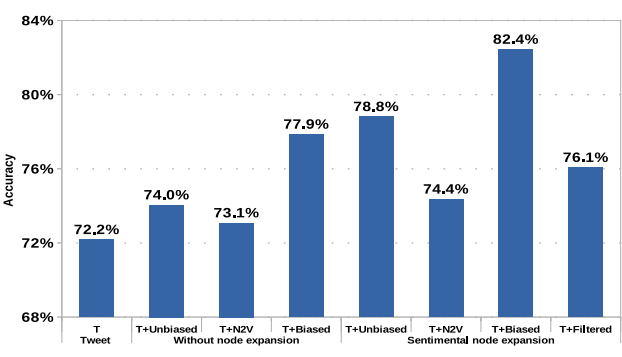

(a) Tweets with keywords $<5$

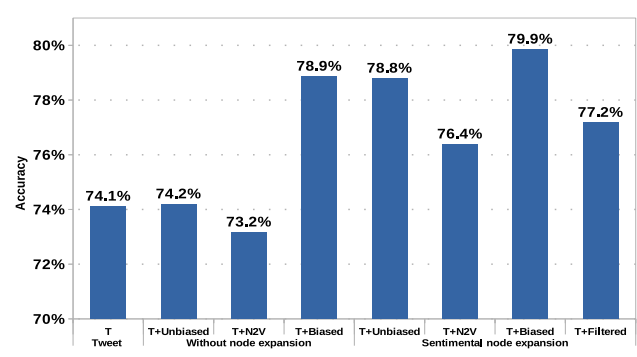

(b) Multilingual tweets

Figure 4: Performance of CNN classifier for different under-specified and multi-lingual tweet categories. Inputs to classifier are 5 different tweet representations; i.e. (i) tweet-text only, and node expansion over the actual tweet using random walkers based on (ii) MNE (Unbiased), (iii) Node2 Vec (N2V), and (iv) centrality biased node expansions (Biased), and (v) random shuffled of the selected sentiment polarized nodes (Filtered).

pansion based representation beats the performance of the tweet representation without any node expansion. Precisely, the sentiment polarized node expansion beats the performance of classifiers with and without non-polarized node expansion by an average margin of $9.19 \%$ and $10.57 \%$, respectively. Further, the non-polarized node expansion beats the performance of the classifiers without node expansion by $1.38 \%$. From Figure 3, we observe two aspects; - i) the expansion of semantically related nodes in tweet-network makes the performance of centrality based biasing algorithm more reliable, ii) the box-plot of sentiment polarized node expansion methods has a small variance, indicating that it is a pretty stable, reliable method to enhance the tweet network view. Hence we can conclude that extending the networked-view of a tweet by including a few semantically similar, central nodes serves our purpose decently. Further, the performance is enhanced in a considerable margin by adding only the sentiment polarized nodes related to the tweet.

\subsection{Response on under-specified Tweets}

We consider tweets having less than five keywords ${ }^{6}$ as an under-specified tweet. Tweets with fewer keywords, although informative, can pose challenges to sentiment classifiers due to under-specificity. We considered the CNN-based classifiers trained using Biased FT embedding to classify the underspecified tweets for this study. Figure 4(a) shows the CNN-based classifiers' performance based on the different types of tweet representations. From the figure, we observed that the sentiment classifier trained without any node expansion performs better than the classifier trained with tweet-text only. This observation shows the power of optimally selected

\footnotetext{
${ }^{6}$ Including hashtags and mentions
}

$n$ random-walk sequences as an alternative representation of tweets. Among no expansion methods, Biased RW sequences give the best performance - beat tweet-text only prediction by $5.7 \%$ and $U n$ biased RW by $3.82 \%$. We can see similar trends of performance for RW based sequences in case of sentiment polarized node expansion also. However, sentiment polarized node expansion strategically mitigates the problem of under-specified tweets by extending the tweet-network view to include less-noisy informative nodes so that the generated walks are more diverse and discriminating. The last pair of columns is one special scenario where we give the original tweet-text + list of randomlyshuffled sentiment polarized nodes to the sentiment classifier. This combination $(\mathrm{T}+$ Filtered $)$ outperforms the tweet only prediction by $3.9 \%$ - depicting nodes selected for expansion are important for inference. However, as T+Biased without node expansion, $\mathrm{T}+$ Unbiased and $\mathrm{T}+$ Biased with sentiment polarized node expansion beat this $\mathrm{T}+$ Filtered by a margin of $1.8 \%, 2.7 \% \& 6.4 \%$ accuracy respectively. This proves the veracity of this fact that random-walk sequences are a stronger representation of tweets as compared to mere inclusion of a shuffled-list of semantically related words to the tweet-text.

\subsection{Response on Multilingual tweet}

Figure 4(b) shows sentiment classification performance over the multilingual tweets - tweet-text written in the code-mixed language. This plot also follows similar trends, as reflected in Figure 4(a), but we have two striking observations this time. In the case of multilingual tweets, since the co-occurrence of multilingual words is rare, our proposed node expansion methods are useful to retrieve semantically related co-occurred English 


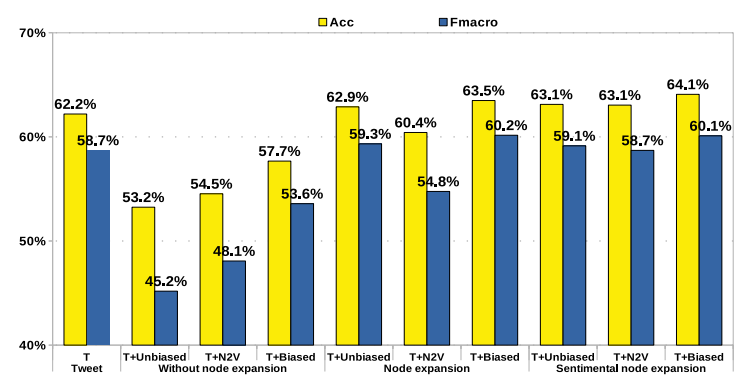

(a) SemEval-2013

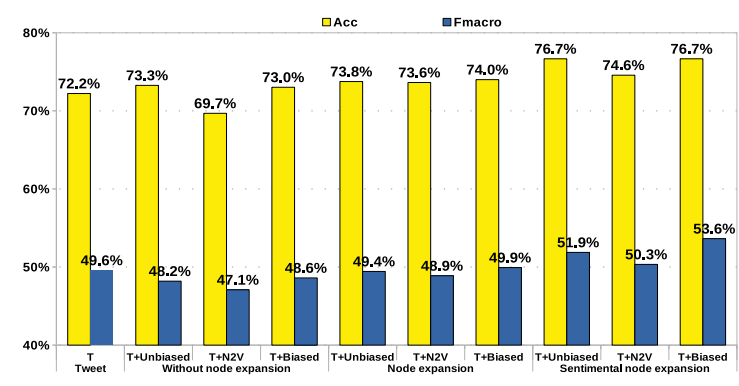

(b) SemEval-2016

Figure 5: Performance of CNN classifiers across SemEval challenge datasets

words that can aid in inference. We verify the same intuition with this plot. We can see the jump in prediction results for sentiment polarized node expansion for $\mathrm{T}+$ Unbiased, $\mathrm{T}+N 2 \mathrm{~V}$, and $\mathrm{T}+$ Biased over their counterparts in the previous group (without node expansion) with a margin of $4.6 \%, 3.2 \%$ and $0.1 \%$ accuracy respectively. It is interesting to see the huge performance improvement of T+Biased without node expansion over tweet only prediction by a margin of $4.75 \%$ accuracy - which we believe is due to the power of interpretable, centrality-score aided, optimally the Biased RW sequences of multilingual words.

\subsection{Evaluation on SemEval datasets}

We further investigate the performance of the proposed method with two popular Twitter datasets used in SemEval challenges for sentiment analysis; SemEval-2013 ${ }^{7}$ and SemEval-2016 ${ }^{8}$. For this study, we consider the train and test split provided in the datasets. Figure 5 (a) and (b) shows the performance of the $\mathrm{CNN}$ classifier trained over different types of tweet representation using the SemEval-2013 and SemEval-2016 datasets, respectively. For training the CNN classifier, we use Biased FT embeddings trained using the challenge datasets. Our proposed centrality aware-based biased random walker through sentiment polarized node expansion has achieved best performance up to $64 \%$ accuracy and $60 \%$ F-macro score on SemEval-2013 and up to $77 \%$ accuracy and $54 \%$ F-macro score for SemEval-2016. Further, on comparing the performance of tweet representation between text-based and network-based without node expansion, it is observed that for both datasets, the representation without node expansion could hardly beat text-based representation in F-macro measure. However, for the SemEval-2016 dataset, our proposed method outperforms text-based representation in both the evaluation measures. We see substantial performance gain for $N 2 V$ RW in both the datasets when augmented with any node expansion. For SemEval-2016, a fascinating thing to observe is - Unbiased and Biased RW-based sequences almost give a comparable performance in terms of accuracy. However, the Biased RW view consistently outperformed the Unbiased view in F-macro measure in both datasets for each of the cases of node expansion. This points to the fact that our method consistently performs better than its counterpart methods.

\section{Conclusion}

This study investigates the efficacy of transforming tweets to heterogeneous multi-layer network for the sentiment classification task. Our proposed centrality aware random-walk method can generate walk sequences that capture better semantic relations than its unbiased and biased random walk based counterparts. From various experimental observations, it is evident that sentiment-oriented node expansion can reduce under-specificity, noise in a tweet, and enhance the representation. The proposed method outperforms its text-based counterpart in a majority of the cases.

\section{Acknowledgments}

We are thankful to Tarun Kumar $^{9}$ for his feedback. This work is partially funded by the Ministry of Electronics \& Information Technology, Government of India.

\footnotetext{
7 https://www.cs.york.ac.uk/semeval-2013/task2/

8 http://saifmohammad.com/WebPages/StanceDataset.
} htm

${ }^{9}$ https://tarunkumariitm.github.io/ 


\section{References}

Nora Al-Twairesh and Hadeel Al-Negheimish. 2019. Surface and deep features ensemble for sentiment analysis of arabic tweets. IEEE Access, 7:8412284131.

Ika Alfina, Dinda Sigmawaty, Fitriasari Nurhidayati, and Achmad Nizar Hidayanto. 2017. Utilizing hashtags for sentiment analysis of tweets in the political domain. In Proceedings of the 9th International Conference on Machine Learning and Computing, pages 43-47. ACM.

Oscar Araque, Ignacio Corcuera-Platas, J Fernando Sánchez-Rada, and Carlos A Iglesias. 2017. Enhancing deep learning sentiment analysis with ensemble techniques in social applications. Expert Systems with Applications, 77:236-246.

AR Balamurali, Aditya Joshi, and Pushpak Bhattacharyya. 2011. Harnessing wordnet senses for supervised sentiment classification. In Proceedings of the 2011 Conference on Empirical Methods in Natural Language Processing, pages 1081-1091. Association for Computational Linguistics.

Luciano Barbosa and Junlan Feng. 2010. Robust sentiment detection on twitter from biased and noisy data. In Proceedings of the 23rd International Conference on Computational Linguistics: Posters, pages 36-44. Association for Computational Linguistics.

Kayvan Bijari, Hadi Zare, Emad Kebriaei, and Hadi Veisi. 2020. Leveraging deep graph-based text representation for sentiment polarity applications. Expert Systems with Applications, 144:113090.

Piotr Bojanowski, Edouard Grave, Armand Joulin, and Tomas Mikolov. 2017. Enriching word vectors with subword information. Transactions of the Association for Computational Linguistics, 5:135-146.

Sergey Brin and Lawrence Page. 1998. The anatomy of a large-scale hypertextual web search engine. Computer Networks, 30:107-117.

Yukuo Cen, Xu Zou, Jianwei Zhang, Hongxia Yang, Jingren Zhou, and Jie Tang. 2019. Representation learning for attributed multiplex heterogeneous network. In Proceedings of the 25th ACM International Conference on Knowledge Discovery \& Data Mining, pages 1358-1368.

Tao Chen, Ruifeng Xu, Yulan He, and Xuan Wang. 2017. Improving sentiment analysis via sentence type classification using bilstm-crf and cnn. Expert Systems with Applications, 72:221-230.

Abdelghani Dahou, Shengwu Xiong, Junwei Zhou, Mohamed Houcine Haddoud, and Pengfei Duan. 2016. Word embeddings and convolutional neural network for arabic sentiment classification. In Proceedings of coling 2016, the 26th international conference on computational linguistics: Technical papers, pages 2418-2427.
Peng Fu, Zheng Lin, Fengcheng Yuan, Weiping Wang, and Dan Meng. 2018. Learning sentiment-specific word embedding via global sentiment representation. In Proceedings of the Thirty-Second AAAI Conference on Artificial Intelligence, (AAAI-18), pages $4808-4815$.

Mahsa Ghorbani, Mahdieh Soleymani Baghshah, and Hamid R Rabiee. 2019. Mgcn: semi-supervised classification in multi-layer graphs with graph convolutional networks. In Proceedings of the 2019 IEEE/ACM International Conference on Advances in Social Networks Analysis and Mining, pages 208211.

Aditya Grover and Jure Leskovec. 2016. node2vec: Scalable feature learning for networks. In Proceedings of the 22nd ACM SIGKDD International Conference on Knowledge Discovery and Data Mining, pages 855-864.

Lin Gui, Yu Zhou, Ruifeng Xu, Yulan He, and Qin Lu. 2017. Learning representations from heterogeneous network for sentiment classification of product reviews. Knowledge-Based Systems, 124:34-45.

Obaida Hanteer and Luca Rossi. 2019. An innovative way to model twitter topic-driven interactions using multiplex networks. Frontiers in Big Data, 2:9.

Zhao Jianqiang, Gui Xiaolin, and Zhang Xuejun. 2018. Deep convolution neural networks for twitter sentiment analysis. IEEE Access, 6:23253-23260.

Mostafa Karamibekr and Ali A Ghorbani. 2012. Sentiment analysis of social issues. In Proceedings of the International Conference on Social Informatics (SocialInformatics), pages 215-221.

Yoon Kim. 2014. Convolutional neural networks for sentence classification. In Proceedings of the 2014 Conference on Empirical Methods in Natural Language Processing, pages 1746-1751. Association for Computational Linguistics.

Mikko Kivelä, Alex Arenas, Marc Barthelemy, James P Gleeson, Yamir Moreno, and Mason A Porter. 2014. Multilayer networks. Journal of complex networks, 2(3):203-271.

Efthymios Kouloumpis, Theresa Wilson, and Johanna D Moore. 2011. Twitter sentiment analysis: The good the bad and the omg! In Proceedings of the International AAAI Conference on Web and Social Media (ICWSM), volume 11, pages 538-541.

John Lafferty and Chengxiang Zhai. 2001. Document language models, query models, and risk minimization for information retrieval. In Proceedings of the 24th annual international ACM SIGIR conference on Research and development in information retrieval, pages 111-119.

Jundong Li, Chen Chen, Hanghang Tong, and Huan Liu. 2018. Multi-layered network embedding. In Proceedings of the 2018 SIAM International Conference on Data Mining, pages 684-692. SIAM. 
Yongjin Li and Jagdish C Patra. 2010. Genomewide inferring gene-phenotype relationship by walking on the heterogeneous network. Bioinformatics, 26(9):1219-1224.

Jiangming Liu and Yue Zhang. 2017. Attention modeling for targeted sentiment. In Proceedings of the 15th Conference of the European Chapter of the Association for Computational Linguistics: Volume 2, Short Papers, pages 572-577.

Dongsheng Luo, Jingchao Ni, Suhang Wang, Yuchen Bian, Xiong Yu, and Xiang Zhang. 2020. Deep multi-graph clustering via attentive cross-graph association. In Proceedings of the 13th International Conference on Web Search and Data Mining, pages 393-401.

Saif Mohammad, Cody Dunne, and Bonnie Dorr. 2009. Generating high-coverage semantic orientation lexicons from overtly marked words and a thesaurus. In Proceedings of the 2009 Conference on Empirical Methods in Natural Language Processing: Volume 2-Volume 2, pages 599-608. Association for Computational Linguistics.

Huy Nguyen and Minh-Le Nguyen. 2018. A deep neural architecture for sentence-level sentiment classification in twitter social networking. In Proceedings of the Computational Linguistics, pages 15-27.

Jingchao Ni, Shiyu Chang, Xiao Liu, Wei Cheng, Haifeng Chen, Dongkuan $\mathrm{Xu}$, and Xiang Zhang. 2018. Co-regularized deep multi-network embedding. In Proceedings of the 2018 World Wide Web Conference, pages 469-478.

Bo Pang, Lillian Lee, and Shivakumar Vaithyanathan. 2002. Thumbs up?: sentiment classification using machine learning techniques. In Proceedings of the ACL-02 Conference on Empirical Methods in Natural Language Processing, volume 10, pages 79-86.

Duc-Hong Pham and Anh-Cuong Le. 2018. Learning multiple layers of knowledge representation for aspect based sentiment analysis. Data \& Knowledge Engineering, 114:26-39.

Rudy Prabowo and Mike Thelwall. 2009. Sentiment analysis: A combined approach. Journal of Informetrics, 3(2):143-157.

Ashequl Qadir and Ellen Riloff. 2013. Bootstrapped learning of emotion hashtags\# hashtags4you. In Proceedings of the 4th workshop on computational approaches to subjectivity, sentiment and social media analysis, pages 2-11.

Ashequl Qadir and Ellen Riloff. 2014. Learning emotion indicators from tweets: Hashtags, hashtag patterns, and phrases. In Proceedings of the 2014 Conference on Empirical Methods in Natural Language Processing, pages 1203-1209.
Meng Qu, Jian Tang, Jingbo Shang, Xiang Ren, Ming Zhang, and Jiawei Han. 2017. An attention-based collaboration framework for multi-view network representation learning. In Proceedings of the 2017 ACM on Conference on Information and Knowledge Management, pages 1767-1776.

Christoph Rahmede, Jacopo Iacovacci, Alex Arenas, and Ginestra Bianconi. 2018. Centralities of nodes and influences of layers in large multiplex networks. Journal of Complex Networks, 6(5):733-752.

Cicero dos Santos and Maira Gatti. 2014. Deep convolutional neural networks for sentiment analysis of short texts. In Proceedings of the 25th International Conference on Computational Linguistics (COLING): Technical Papers, pages 69-78.

Aliaksei Severyn and Alessandro Moschitti. 2015. Twitter sentiment analysis with deep convolutional neural networks. In Proceedings of the 38th International ACM SIGIR Conference on Research and Development in Information Retrieval, pages 959-962.

L. G. Singh, A. Anil, and S. R. Singh. 2020. She: Sentiment hashtag embedding through multitask learning. IEEE Transactions on Computational Social Systems, 7(2):417-424.

Maite Taboada, Julian Brooke, Milan Tofiloski, Kimberly Voll, and Manfred Stede. 2011. Lexicon-based methods for sentiment analysis. Computational linguistics, 37(2):267-307.

Duyu Tang, Furu Wei, Bing Qin, Nan Yang, Ting Liu, and Ming Zhou. 2016. Sentiment embeddings with applications to sentiment analysis. IEEE Transactions on Knowledge and Data Engineering, 28(2):496-509.

Peter D Turney. 2002. Thumbs up or thumbs down?: semantic orientation applied to unsupervised classification of reviews. In Proceedings of the 40th annual meeting on Association for Computational Linguistics, pages 417-424. Association for Computational Linguistics.

Peter D Turney and Michael L Littman. 2003. Measuring praise and criticism: Inference of semantic orientation from association. ACM Transactions on Information Systems (TOIS), 21(4):315-346.

John Violos, Konstantinos Tserpes, Evangelos Psomakelis, Konstantinos Psychas, and Theodora Varvarigou. 2016. Sentiment analysis using wordgraphs. In Proceedings of the 6th International Conference on Web Intelligence, Mining and Semantics, pages 1-9.

Gang Wang, Jianshan Sun, Jian Ma, Kaiquan $\mathrm{Xu}$, and Jibao Gu. 2014. Sentiment classification: The contribution of ensemble learning. Decision support systems, 57:77-93. 
Xiaolong Wang, Furu Wei, Xiaohua Liu, Ming Zhou, and Ming Zhang. 2011. Topic sentiment analysis in twitter: a graph-based hashtag sentiment classification approach. In Proceedings of the 20th ACM International Conference on Information and Knowledge Management, pages 1031-1040. ACM.

Yuan Wang, Jie Liu, Yalou Huang, and Xia Feng. 2016. Using hashtag graph-based topic model to connect semantically-related words without co-occurrence in microblogs. IEEE Transactions on Knowledge and Data Engineering, 28(7):1919-1933.

Jason Weston, Sumit Chopra, and Keith Adams. 2014. \# tagspace: Semantic embeddings from hashtags. In Proceedings of the 2014 Conference on Empirical Methods in Natural Language Processing, pages 1822-1827.

Guixian Xu, Yueting Meng, Xiaoyu Qiu, Ziheng Yu, and $\mathrm{Xu} \mathrm{Wu}$. 2019. Sentiment analysis of comment texts based on bilstm. IEEE Access, 7:5152251532 .

Hongming Zhang, Liwei Qiu, Lingling Yi, and Yangqiu Song. 2018. Scalable multiplex network embedding. In IJCAI, volume 18, pages 3082-3088.

Zhou Zhao, Hanqing Lu, Deng Cai, Xiaofei He, and Yueting Zhuang. 2017. Microblog sentiment classification via recurrent random walk network learning. In IJCAI, volume 17, pages 3532-3538.

Guang-You Zhou and Jimmy Xiangji Huang. 2017. Modeling and mining domain shared knowledge for sentiment analysis. ACM Transactions on Information Systems (TOIS), 36(2):1-36.

Marinka Zitnik and Jure Leskovec. 2017. Predicting multicellular function through multi-layer tissue networks. Bioinformatics, 33(14):i190-i198. 


\section{A Appendix}

Here, we show some additional experiment results and their implications in support of our proposed framework for sentiment classification.

\section{A.1 Interpretation of node centrality scores and layer influences}

In Table 4 and Figure 8 , we precisely show three example tweets and their ranked centrality scores calculated by our proposed method. The first example is all about a terrorist attack in India and India's Prime Minister Modi's reaction to it. In simple multi-layer view of a tweet, we see india, pm, modi, speech - keywords related to how India reacts have more centrality than the attack \#uriattack and one terrorist named \#burhanwani. It is interesting to look at the list of nodes selected by our plain and sentiment polarized node expansion methods in Table 4. The list of nodes for expansion related to \#uriattack talk about the surgical strike, home minister, defense minister, soldiers killed in this attack, and have higher ranks. The second tweet is one under-specified tweet where India's Prime Minister greets soldiers. Here, our node expansion methods beautifully guess that this greeting is related to India's success in \#surgicalstrike as India's reaction to \#uriattack. Keywords related to the war, causalities and related emotions like army, pak, loc, diplomatic, refute, lose, collateral, pray, roar come higher in centrality-score based ranking. Example 3 is one multilingual tweet whose main theme is Goods \& Services Tax (GST) (a bill related to tax payment adopted by the Indian government in 2017). Although the original tweet mentions @ narendramodi PM of India and uses Hindi keywords, but the nodes selected for expansion rightfully capture about finance ministry (@arunjaitley, @finminindia),home ministry (@amitshah), economic transformation and mostly positive sentiments about it. Also, as we create one large multi-layer heterogeneous network from the tweet corpus to train node embedding methods, the layer influence calculated by our method ranks the hashtag layer higher than the mention layer followed by the keyword layer $(H>M>K)$. This ranking is pretty intuitive as we have most of the influential nodes in the hashtag (trending topics) and mention (Twitter handles of important personalities) networks. Whereas, the keyword layer has a large number of keywords, among them, the entire population of the less frequently used

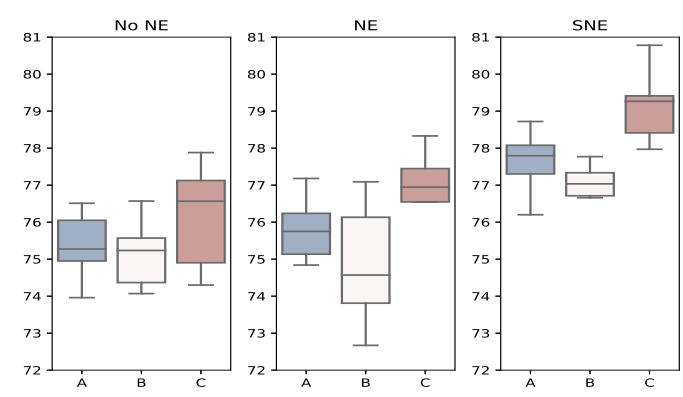

Figure 6: Effectiveness of centrality score-based biased representation of tweets A:Unbiased, B:Node2Vec, C:Biased representation of networked-tweets for No Node Expansion(No $\mathrm{NE}$ ), Node Expansion(NE), sentiment polarized node expansion (SNE) methods. Accuracy(\%) of sentiment prediction in Y-axis.

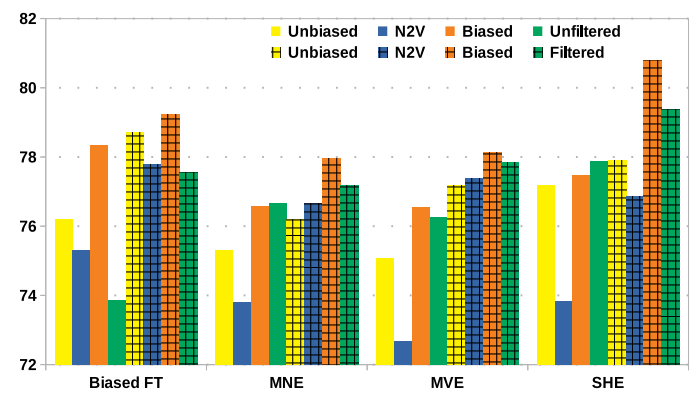

Figure 7: Effectiveness of (sentiment polarized) node expansion in tweet-networks. Patterned and plain colored bars shows the performance with and without sentiment polarized node expansion respectively.

keywords brings down the overall influence score of this layer.

\section{A.2 Novelty of centrality score-based tweet network representation}

We created boxplots of aggregated performances of three competing methods (Unbiased, Node2 Vec and Biased as in Table 3) for tweet network representation and generation of RW sequences. From Figure 6, for each networked view of tweets (NE, No NE, SNE), it is evident that our centrality scorebased RW sequences provide better tweet representations than unbiased and Node2 Vec biasing based RW sequences. Node2Vec biasing does not seem to be an intuitive tweet networked view for tweet sentiment classification. Our proposed centrality aware RW sequences beat Node2 Vec by $3.1 \%$ and unbiased RWs by $1.7 \%$ on average.

\section{A.3 Novelty of (sentiment polarized) node expansion: more insights}

In Figure 7, we compare two node expansion methods that we propose as part of our framework. The 


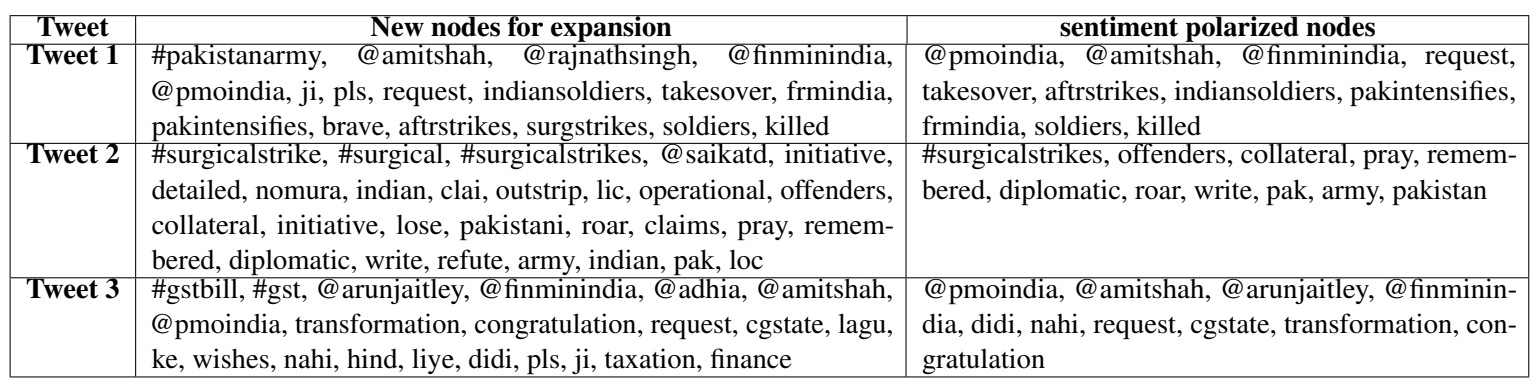

Table 4: Nodes selected for tweet view expansion. Tweet 1: @asadmunir38 Modi is agressive since \#UriAttack, \#BurhanWani \& PM speech@UNGAPakneeds to start dialogue with neighbours India, Afghan; Tweet 2: @ narendramodi \#GreetingsToSoldiers; Tweet 3: @narendramodi Thank you Sir GST laagu karne ke liye is India great

patterned and plain colored bars show the performance with and without sentiment polarized node expansion, respectively. Evidently, sentiment polarized node expansion offers a performance improvement of $8.4 \%$ over non-polarized node expansion on average across all four embedding methods. Even sentiment polarized node expansion improves the tweet representation with a list of unfiltered nodes without any network structure by an accuracy improvement of $1.8 \%$ on average - which clearly shows that list of nodes selected for sentiment polarized node expansion are less-noisy and informative in the context of tweet sentiment classification. 
A

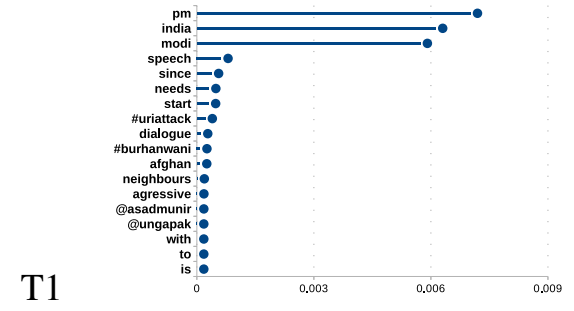

$\mathrm{T} 2$

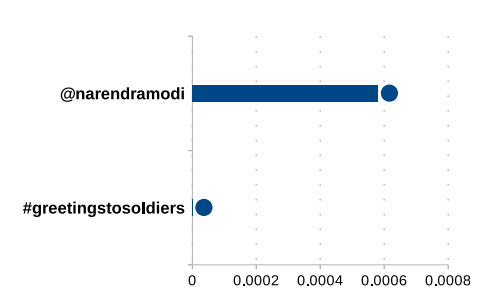

$\mathrm{T} 3$
B
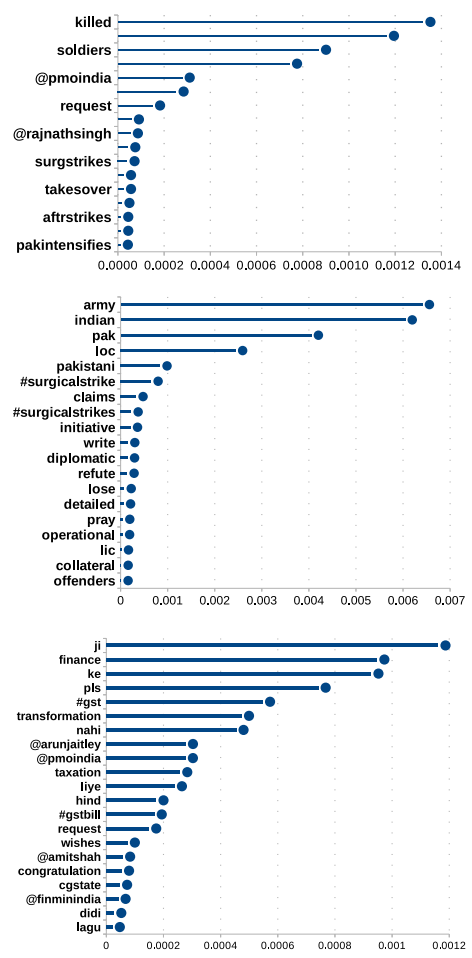

$\mathrm{C}$
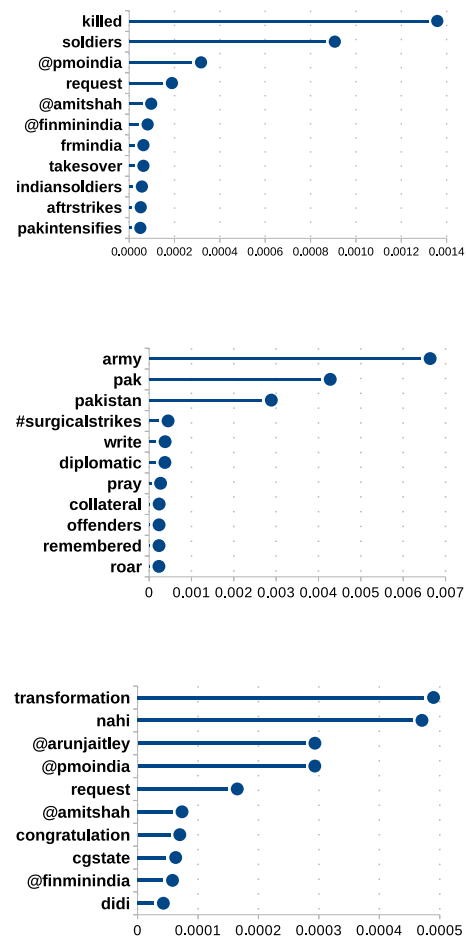

Figure 8: Tweet component centrality rankings A: without node expansion, B: plain node expansion, C: sentiment polarized node expansion. T1: @asadmunir38 Modi is agressive since \#UriAttack, \#BurhanWani \& PM speech @UNGAPak needs to start dialogue with neighbours India, Afghan; T2: @narendramodi \#GreetingsToSoldiers; T3: @ narendramodi Thank you Sir GST laagu karne ke liye is India great. 